\title{
Simulation numérique de la coupe orthogonale par couplage thermique-comportement-endommagement en transformations finies
}

\author{
Philippe Lestriez ${ }^{1,2, a}$, Khémais SaAnouni ${ }^{1}$ et Abel Cherouat ${ }^{1}$ \\ 1 LASMIS-GSM, U.T.T., 12 rue Marie Curie, 10010 Troyes Cedex, France \\ 2 Structures et Matériaux, E.S.T.A.C.A., 34 rue Victor Hugo, 92300 Levallois-Perret, France
}

Reçu le 25 février 2005, accepté le 21 mars 2005

\begin{abstract}
Résumé - Ce travail est consacré à la présentation d'une nouvelle méthodologie de prévision de l'usinage par enlèvement de copeau. Cette méthodologie est basée sur le couplage fort entre le comportement thermo-mécanique et l'endommagement ductile. Elle utilise également une adaptation de la taille des éléments en fonction de critères physiques (endommagement, plasticité, ...) et géométriques (courbures locales, ... ). Cette approche est étudiée et comparée à l'approche classique basée sur le simple écoulement visco-plastique.

Mots clés : Élasto-visco-plasticité / endommagement / thermique / couplage fort / coupe orthogonale / copeaux / ABAQUS/EXPLICIT

Abstract - Numerical simulation of the orthogonal cutting, using a finite strain fully coupled thermo-mechanical equations with ductile damage. This work is devoted to the study of a new numerical methodology for the simulation of any metal machining by chip formation leading to a very useful virtual machining tool. This methodology is based on the strong coupling between the thermomechanical behaviour and the ductile damage together with adaptive meshing facility based on some physical (damage, plastic strain, ...) or geometrical (tools local curvature, ...) criteria. This approach is studied and compared with the traditional approach based on the simple viscoplastic flow in the case of orthogonal cutting operation.
\end{abstract}

Key words: Elasto-visco-plasticity / ductile damage / thermal effect / strong coupling / metal chip / ABAQUS/EXPLICIT

\section{Introduction}

L'usinage des métaux est une technique de mise en forme par enlèvement de matière, qui bien que très développée et très répandue du point de vue pratique, n'est pas encore maîtrisée du point de vue modélisation théorique et simulation numérique. C'est l'empirisme qui est largement en vigueur dans ce domaine où l'on procède encore par erreur-correction. En effet, la coupe des métaux fait intervenir de très nombreux phénomènes mécaniques, thermiques et métallurgiques qui doivent être pris en considération pour simuler numériquement ce type de procédé.

Plusieurs travaux dédiés à la simulation numérique des procédés d'usinage par enlèvement de matière se sont développés ces dernières années. Les modèles développés ont permis aussi de prendre en compte plusieurs phénomènes physiques très présents lors de la coupe

\footnotetext{
a Auteur correspondant : plestriez@estaca.fr
}

tels que les grandes vitesses de déformation plastique, la génération de chaleur, le frottement et le contact. Ceci se fait moyennant l'utilisation de formulations par éléments finis faisant appel ou non à un critère de séparation du copeau de la pièce et des procédures et lois de comportement, assurant un couplage plus ou moins fort entre phénomènes mécaniques et thermiques. On distingue deux types de modélisation pour reproduire la formation de copeau :

- Les modèles basés sur l'écoulement viscoplastique de la matière sur les faces de coupe de l'outil. Dans ces modèles, on n'impose ni un chemin ni un critère de séparation du copeau. Il suffit d'imposer des conditions aux limites sur la pièce, sur la géométrie du copeau dans certaines formulations et sur la trajectoire de l'outil. La formation de copeau se fait par écoulement visco-plastique de la matière sur la face de coupe de l'outil. C'est de loin l'approche numérique la plus utilisée dans la littérature [1-3]. 
- Les modèles basés sur un critère de rupture pour simuler la formation de copeau. En général deux méthodologies sont utilisées :

- Les méthodes utilisant la technique de relâchement des nœuds devant la pointe de l'outil afin de former le copeau sur la base d'un critère géométrique ou physique. Ces critères consistent en premier lieu à fixer une ligne ou un chemin décrit par des nœuds dédoublés qui vont se détacher au fur et à mesure que l'outil avance. Ce critère a été utilisé dans plusieurs études telles que [4-6].

- Les méthodes utilisant la suppression d'éléments sur la base de critères locaux (plasticité, contrainte max, endommagement, ....). Au fur et à mesure que l'outil avance, la fissure est initiée et propagée dans les éléments en contact direct avec le bec de l'outil, une fois que le critère local atteint une valeur critique qu'on se fixe. Ces éléments seront éliminés pour laisser avancer l'outil. D'autres modèles utilisent la déformation plastique ou la contrainte équivalente dans l'élément comme critère d'élimination [7-9].

Dans ce travail, une nouvelle méthodologie numérique de simulation de la coupe est présentée. Basée sur un modèle couplant les différents champs thermomécaniques à l'endommagement ductile, cette méthodologie permet de reproduire assez fidèlement la formation de copeau incluant sa possible segmentation.

Les aspects théoriques et numériques sont tout d'abord explicités en précisant les schémas de résolution dynamique explicite et d'intégration locale du modèle couplé. L'application est ensuite faite à la simulation de la coupe orthogonale. Une attention particulière est accordée à la comparaison entre la méthode sans endommagement et celle prenant en compte le couplage comportement endommagement.

\section{Modélisation}

Dans cette section, les principaux aspects du modèle entièrement couplé sont brièvement rappelés aussi bien d'un point théorique que numérique. La résolution $\mathrm{du}$ problème décrivant les grandes déformations du métal pendant le processus de formation est régie par deux équations aux dérivées partielles entièrement couplées : l'équilibre mécanique et les équations thermiques [10]. Les équations thermiques résultent du principe de la conservation d'énergie décrivant la distribution de la température en fonction de la dissipation mécanique (plasticité, écrouissages et endommagement). Les équations mécaniques ne sont autres que les équations d'équilibre qui dérivent du principe des puissances virtuelles. Grâce à ces équations, en utilisant la méthode des éléments finis en déplacement et en température (Galerkin), on obtient après assemblage le système algébrique non-linéaire suivant :

$$
\left\{\begin{array}{l}
{[M]\{\ddot{u}\}-\{F(u, T)\}=0} \\
{[\Theta]\{\dot{T}\}+\left[K^{\mathrm{Th}}\right]\{T\}-\left\{R^{\text {Ther }}(u, T)\right\}=0}
\end{array}\right.
$$

avec $[M]$ la matrice masse, $[\theta]$ et $\left[K^{\mathrm{Th}}\right]$ les matrices thermiques définies par :

$$
\begin{aligned}
& {[M]=\sum_{j} \int_{V^{j}} \rho\left[N^{k}\right]^{T}\left[N^{i}\right] \mathrm{d} V} \\
& {[\Theta]=\sum_{j} \int_{V^{j}} \rho C_{v}\left[N^{k}\right]^{T}\left[N^{i}\right] \mathrm{d} V} \\
& {\left[K^{\mathrm{Th}}\right]=-\int_{V^{j}}(k T)\left[B_{T}^{k}\right]^{T}\left[B_{T}^{k}\right] \mathrm{d} V}
\end{aligned}
$$

$\{F\}$ est le vecteur des sollicitations mécaniques et $\left\{R^{\text {Ther }}\right\}$ le vecteur des sollicitations thermiques donnés par:

$$
\begin{aligned}
& \{F(u, T)\}= \\
& \sum_{j}\left\{\begin{array}{l}
-\int_{V^{j}}\left[B^{k}\right]^{T}\{\underline{\sigma}\} \mathrm{d} V+\int_{V^{j}}\left[N^{k}\right]^{T}\left\{f_{\mathrm{d}}\right\} \mathrm{d} V \\
+\int_{S_{F}^{j}}\left[N^{k}\right]^{T}\{t\} \mathrm{d} S+\int_{S_{c}^{j}}\left[N^{k}\right]^{T}\left[t_{\mathrm{c}}\right] \mathrm{d} S
\end{array}\right\} \\
& \left\{R^{\text {Ther }}(u, T)\right\}= \\
& \sum_{j}\left\{\begin{array}{l}
-\int_{V^{j}}\left[N_{T}^{k}\right]^{T}\{\pi\} \mathrm{d} V+\int_{V^{j}}\left[N_{T}^{k}\right]^{T}\left\{R_{\mathrm{pl}}\right\} \mathrm{d} V \\
-\int_{S_{\bar{q}}^{j}}\left[N_{T}^{k}\right]^{T}\{\bar{q}\} \mathrm{d} S+\int_{S_{T}^{j}}\left[N_{T}^{k}\right]\left\{q_{\mathrm{f}}\right\} \mathrm{d} S
\end{array}\right\} \\
& R_{\mathrm{pl}}=\underline{\sigma}: \underline{\dot{\varepsilon}}^{\mathrm{p}}-\underline{X}: \underline{\dot{\alpha}}-R \dot{r}+Y \dot{D} \\
& +T\left(\frac{\partial \underline{\sigma}}{\partial T}: \underline{\dot{\varepsilon}}^{\mathrm{e}}-\frac{\partial Y}{\partial T} \dot{D}+\frac{\partial \underline{X}}{\partial T}: \underline{\dot{\alpha}}+\frac{\partial R}{\partial T} \dot{r}\right)
\end{aligned}
$$

Dans ces équations $\{\ddot{u}\}$ est le vecteur d'accélération nodal, $\{u\}$ le vecteur déplacement, $\{\dot{T}\}$ est le vecteur flux de température et $\{T\}$ le vecteur de température nodal. Le vecteur $\left\{f_{\mathrm{d}}\right\}$ représente les forces de volume, le vecteur $\{t\}$, les forces surfaciques et le vecteur $\left\{t_{c}\right\}$, les forces de contact, le vecteur $\{\pi\}$, la densité volumique de production de chaleur, le vecteur $\{\bar{q}\}$, le flux de chaleur imposé par les conditions aux limites de type Neumann et enfin le vecteur $\left\{q_{\mathrm{f}}\right\}$, le flux de chaleur inconnu correspondant au champ de température connu sur la frontière $S_{T}$ donné conformément à la loi de Fourier. Les matrices $[N]$ et $\left[N_{T}\right]$ définissent respectivement les fonctions d'interpolation du déplacement et de la température, $[B]$ et $\left[B_{T}\right]$ leurs dérivés spatiales. $C_{v}$ est la chaleur spécifique, $k$ le coefficient de conduction et $\rho$ est la densité du matériau.

Les différentes variables sont :

- $\left(\underline{\sigma}, \underline{D}^{\mathrm{p}}\right)$ la contrainte de Cauchy et le tenseur du taux de déformation plastique assumant $\underline{D}=\underline{\dot{\varepsilon}}_{j}^{\mathrm{e}}+\underline{D}^{\mathrm{p}}$ où $\underline{D}$ est la taux de déformation totale et $\underline{\varepsilon}_{j}^{\mathrm{e}}$ est le taux de déformation élastique selon Jaumann. 
- $(\underline{X}, \underline{\dot{\alpha}})$ les tenseurs déviatoriques représentant l'écrouissage cinématique.

- $(R, \dot{r})$ les variables scalaires représentant l'écrouissage isotrope.

- $(Y, \dot{D})$ les variables scalaires représentant l'endommagement ductile.

Pour résoudre le premier système en ce qui concerne le déplacement $(u, v, w)$ et la température $(T)$, des schémas explicites de résolution sont employés en utilisant le logiciel ABAQUS (voir les manuels utilisateurs ABAQUS/EXPLICIT). La résolution se fait séquentiellement sur l'intervalle de temps $\left[t_{n}, t_{n+1}=t_{n}+\Delta t\right]$ selon le premier système, la démarche suivante est utilisée :

- Résolution du problème mécanique avec une température constante $\left\{T_{n}\right\}$

$$
\begin{aligned}
& \left\{\ddot{u}_{n}\right\}=[M]^{-1}\left\{F\left(u_{n}, T_{n}\right)\right\} \\
& \left\{\dot{u}_{n+\frac{1}{2}}\right\}=\left\{\dot{u}_{n-\frac{1}{2}}\right\}+\frac{\Delta t_{n+1}+\Delta t_{n}}{2}\left\{\ddot{u}_{n}\right\} \\
& \left\{u_{n+1}\right\}=\left\{u_{n}\right\}+\Delta t_{n+1}\left\{\dot{u}_{n+\frac{1}{2}}\right\}
\end{aligned}
$$

- Résolution du problème thermique par rapport à $\left\{T_{n+1}\right\}$ connaissant les différents champs mécaniques (contraintes, écrouissages, endommagement)

$$
\begin{aligned}
& \left\{\dot{T}_{n}\right\}=[\Theta]^{-1}\left\{R^{\text {Ther }}\left(u_{n+1}, T_{n}\right)\right\} \\
& \left\{T_{n+1}\right\}=\left\{T_{n}\right\}+\Delta t_{n}\left\{\dot{T}_{n}\right\}
\end{aligned}
$$

Cette stratégie explicite de résolution a besoin d'une routine utilisateur Vumat (voir les manuels utilisateurs ABAQUS/EXPLICIT) pour calculer $\sigma_{n+1}$ et $t_{c_{n+1}}$ afin d'évaluer $\{F(u, T)\}$ et pour calculer la valeur de la source interne de chaleur provenant de la mécanique $R_{\mathrm{pl}}$ afin d'évaluer $\left\{R^{\text {Ther }}(u, T)\right\}$.

Pour le problème mécanique, le calcul des forces de contact $\left\{t_{\mathrm{c}}\right\}$ est effectué en employant le modèle de frottement de type Coulomb disponible dans ABAQUS et défini par le coefficient de frottement $\eta$ (voir les manuels utilisateurs ABAQUS/EXPLICIT). Le calcul des différents champs mécaniques est réalisé en intégrant numériquement les équations différentielles ordinaires suivantes (modèle de comportement) [11,12] :

- Pour le champ plastique

$$
\left\{\begin{array}{l}
\underline{\sigma}=(1-D)\left[\lambda_{\mathrm{e}}\left(\underline{\varepsilon}^{\mathrm{e}}: \underline{1}\right) \underline{1}+2 \mu \underline{\varepsilon}^{\mathrm{e}}-3 K \zeta \frac{\left(T-T_{0}\right)}{\sqrt{1-D}} \underline{1}\right] \\
\underline{D}^{\mathrm{p}}=\dot{\lambda} \frac{3}{2} \frac{1}{\sqrt{1-D}} \frac{\underline{S}-\underline{X}}{J_{2}(\underline{\sigma}-\underline{X})}=\dot{\lambda} \underline{n}
\end{array}\right.
$$

- Pour l'écrouissage isotrope

$$
\left\{\begin{array}{l}
R=Q(1-D) r \\
\dot{r}=\dot{\lambda}\left(\frac{1}{\sqrt{1-D}}-b r\right)
\end{array}\right.
$$

- Pour l'écrouissage cinématique

$$
\left\{\begin{array}{l}
\underline{X}=\frac{2}{3} C(1-D) \underline{\alpha} \\
\underline{\dot{\alpha}}=\dot{\lambda}(\underline{n}-a \underline{\alpha})=\underline{\dot{\varepsilon}}^{\mathrm{p}}-a \dot{\lambda} \underline{\alpha}
\end{array}\right.
$$

- Pour l'endommagement ductile

$$
\left\{\begin{array}{l}
\dot{D}=\frac{\dot{\lambda}}{(1-D)^{\beta}}\left[\frac{Y-Y_{0}}{S}\right]^{\mathrm{s}} \\
Y=\frac{1}{2} \lambda_{\mathrm{e}}\left(\underline{\varepsilon}^{\mathrm{e}}: \underline{1}\right)^{2}+\mu \underline{\varepsilon}^{\mathrm{e}}: \underline{\varepsilon}^{\mathrm{e}} \\
-\frac{3 K \zeta}{2 \sqrt{1-D}}\left(T-T_{0}\right) \underline{\varepsilon}^{\mathrm{e}}: \underline{1}+\frac{2}{3} C \underline{\alpha}: \underline{\alpha}+\frac{1}{2} Q r^{2}
\end{array}\right.
$$

avec $K$ le module de compressibilité $\left(K=\left(3 \lambda_{\mathrm{e}}+\right.\right.$ $2 \mu) / 3$ ), $Q$ et $C$ sont les modules d'écrouissage isotrope et cinématique, $a$ et $b$ les coefficients correspondant aux non-linéarités, $\lambda_{\mathrm{e}}$ et $\mu$ sont les coefficients de Lamé, $\zeta$ est le coefficient de dilation thermique, $T_{0}$ la température de référence et $s, S, Y_{0}$ et $\beta$ sont les paramètres matériau caractérisant l'évolution de l'endommagement ductile. Tous les paramètres peuvent être dépendants de la température.

Dans le cas où l'écoulement plasticité dépend du temps (viscoplasticité), le multiplicateur de Lagrange $\dot{\lambda}$ s'écrit :

$$
\dot{\lambda}=\left\langle\frac{\frac{|\underline{\sigma}-\underline{X}|-R}{\sqrt{1-D}}-\sigma_{y}}{\kappa}\right\rangle
$$

où $\kappa$ et $n$ sont les paramètres classiques de viscosité dépendant de la température.

L'intégration numérique de ce système est exécutée par une résolution implicite après avoir réalisé une réduction du nombre d'équation [9]. Notons simplement que l'implémentation de ce modèle dans ABAQUS via la routine VUMAT a été faite de telle sorte que le calcul peut se faire soit avec couplage comportementendommagement $\left(k_{\text {couple }}=1\right)$ soit sans couplage $\left(k_{\text {couple }}=0\right)$. Dans ce dernier cas, l'effet de l'endommagement sur le comportement n'est pas pris en compte. Par ailleurs la création de fissures par suppression d'éléments totalement endommagés, nous conduit à utiliser une méthode d'adaptation de maillage sur des critères physiques et géométriques $[9,13,14]$. Ceci permet de raffiner au maximum la taille des éléments se trouvant dans les zones à fort endommagement $\left(h_{\min }\right.$ est la taille minimale des éléments).

\section{Simulation de la coupe orthogonale}

L'objectif ici est d'appliquer la modélisation du couplage multi-physique développée ci-dessus pour voir si elle est potentiellement capable de simuler les opérations de coupe orthogonale. 
Tableau 1. Propriétés du matériau à différentes températures.

\begin{tabular}{ccccccc}
\hline Coefficients du modèle & \multicolumn{5}{c}{ Températures } \\
\hline & & $20{ }^{\circ} \mathrm{C}$ & $200{ }^{\circ} \mathrm{C}$ & $250{ }^{\circ} \mathrm{C}$ & $280{ }^{\circ} \mathrm{C}$ & $320{ }^{\circ} \mathrm{C}$ \\
\hline \multirow{2}{*}{ Paramètres d'élasticité } & $E(\mathrm{MPa})$ & 72615 & 66000 & 61500 & 58000 & 56000 \\
& $\nu$ & 0,3 & 0,3 & 0,3 & 0,3 & 0,3 \\
& $\sigma_{y}(\mathrm{MPa})$ & 97 & 93 & 81 & 68 & 44 \\
\hline \multirow{2}{*}{ Paramètres de viscosité } & $K_{\text {visco }}(\mathrm{MPa})$ & 130 & 115 & 90 & 85 & 85 \\
& $n$ & 4,22 & 4,22 & 4,22 & 4,22 & 4,22 \\
\hline \multirow{2}{*}{ Paramètres de l'écrouissage isotrope } & $Q(\mathrm{MPa})$ & 5850 & 3700 & -60 & -85 & -85 \\
& $b$ & 40 & 35 & 5 & 3 & 1 \\
\hline \multirow{2}{*}{ Paramètres de l'écrouissage cinématique } & $C(\mathrm{MPa})$ & 42000 & 25000 & 11600 & 9300 & 4000 \\
& $a$ & 448 & 445 & 220 & 205 & 200 \\
\hline \multirow{2}{*}{ Paramètres de l'endommagement du matériau } & $\beta$ & 25 & 23 & 12,5 & 7,5 & 4,6 \\
& $S(\mathrm{MPa})$ & 1,13 & 0,86 & 0,69 & 0,59 & 0,55 \\
& $Y_{0}(\mathrm{MPa})$ & 0,44 & 0,4 & 0,17 & 0,115 & 0,0445 \\
\hline
\end{tabular}

Le comportement du matériau est de type viscoplastique où les différents paramètres dépendent de la température. Plusieurs études vont être présentées. Tout d'abord nous avons réalisé des essais avec un modèle thermo-élasto-visco-plastique sans endommagement (le plus souvent utilisé dans la littérature). Avec ce modèle nous avons fait varier les paramètres tels que la profondeur de passe $(p)$, l'angle de coupe $(\gamma)$, l'angle de dépouille $(\alpha)$, et enfin la vitesse de coupe $\left(V_{\mathrm{c}}\right)$, pour étudier leur influence respective sur la distribution de la température et des efforts de coupe. Ensuite, nous avons utilisé le modèle thermo-élasto-visco-plastique avec endommagement pour différentes ductilités. Ceci permettra une comparaison objective entre les deux méthodologies.

Le matériau utilisé est un alliage d'aluminium AS7U3G T5 [15]. Suite à des essais de traction effectués à différentes températures, nous avons fait une identification sur un point de Gauss avec le logiciel ZEBULON 8,2 puis avec ABAQUS/EXPLICIT sur une éprouvette de traction. Nous avons, pour plusieurs températures, obtenu les paramètres cités dans le tableau 1 .

Comme la plupart de ces paramètres évoluent en fonction de la température, on se propose alors de déterminer pour chacun une loi d'évolution. Ces fonctions sont introduites dans la routine VUMAT. Pour chaque paramètre identifié, nous avons fixé une température maximale (qui varie entre 350 et $420{ }^{\circ} \mathrm{C}$ ) au-delà de laquelle il n'y a plus d'évolution puisque le matériau est supposé devenu en état liquide.

Pour un paramètre $P \in\left\{E, \sigma_{y}, K, n, Q, b, C, a, s\right.$, $\left.S, Y_{0}, \beta\right\}$, l'expression de sa loi d'évolution en fonction de la température est :

$$
\begin{aligned}
P=\frac{1}{2}\left[\left(P_{1}-P_{2}\right)\right. & \left(\left(1-e^{-P_{3} \frac{\left\langle T_{\mathrm{r}}-T\right\rangle}{T_{\mathrm{F}}-T_{\mathrm{r}}}}\right)\right. \\
& \left.\left.+\left(e^{-P 4 \frac{\left\langle T-T_{\mathrm{r}}\right\rangle}{T_{\mathrm{F}}-T_{\mathrm{r}}}}\right)\right)+2 P_{2}\right]
\end{aligned}
$$

avec $P_{1}, P_{2}, P_{3}$ et $P_{4}$ définis comme des paramètres de la loi d'évolution qui permettent de bien caler la courbe théorique avec les valeurs identifiées. $T_{\mathrm{f}}$ est la température de fusion du matériau et $T_{\mathrm{r}}$ la température de référence de changement de concavité. Par ailleurs, nous avons supposé que $\nu=0,3$ et $n=4,22$ ne sont pas variables en fonction de la température pour le matériau étudié. Le tableau 2 résume les valeurs de tous les paramètres intervenant dans l'équation précédente et ce pour tous les coefficients variables avec la température. Notons que pour l'alliage AS7U3G, nous avons pris $T_{\mathrm{f}}=600{ }^{\circ} \mathrm{C}$ et $T_{\mathrm{r}}=220^{\circ} \mathrm{C}$.

Connaissant les différents paramètres du matériau, nous avons ensuite simulé plusieurs essais de coupe orthogonale. La chaleur spécifique prise est $C_{v}=$ $880 \mathrm{~J} \cdot \mathrm{kg}^{-1} \cdot{ }^{\circ} \mathrm{C}$, le coefficient de conductivité $k=$ $38 \mathrm{~W} \cdot \mathrm{m}^{-1} \cdot{ }^{\circ} \mathrm{C}$, le coefficient de dilatation linéique thermique $\zeta=10^{-5}{ }^{\circ} \mathrm{C}^{-1}$ et la masse volumique $\rho=$ $2796 \mathrm{~kg} . \mathrm{m}^{-3}$. La pièce à découper est de longueur $50 \mathrm{~mm}$ et de largeur $30 \mathrm{~mm}, p$ représente la profondeur de passe, $\gamma$ l'angle d'attaque, $\alpha$ l'angle de dépouille et $V_{\mathrm{c}}$ la vitesse d'avance de l'outil. Le rayon de bec de l'outil est de 0,1 mm. L'élément utilisé est le CPE3RT de la librairie d'ABAQUS. La taille de maille utilisée lors de l'identification sur ABAQUS étant de $0,2 \mathrm{~mm}$, la taille retenue pour les essais a été $h_{\min }=0,2 \mathrm{~mm}$ pour la taille minimale et $h_{\max }=4 \mathrm{~mm}$ pour la taille maximale. La pièce est encastrée sur sa partie inférieure et a pour température initiale $20^{\circ} \mathrm{C}$. Le coefficient de frottement de la loi de Coulomb entre la pièce et les outils est supposé constant (indépendant de la température!) de valeur $\mu=0,3$.

\subsection{Calculs sans couplage comportement-endommagement}

Comme nous l'avons déjà signalé, plusieurs auteurs simulent la formation de copeaux par simple écoulement visco-plastique de la matière sur la face de coupe de l'outil. Ainsi, cela s'apparente à l'enfoncement d'un pieu (l'outil) dans un massif (la pièce) supposé thermo-viscoplastique. Plus rares sont les méthodes qui considèrent 
Tableau 2. Propriétés du matériau à différentes températures.

\begin{tabular}{cccccc}
\hline Coefficients du modèle & & $P_{1}$ & $P_{2}$ & $P_{3}$ & $P_{4}$ \\
\hline \multirow{2}{*}{ Paramètres d'élasticité } & $E(\mathrm{MPa})$ & 72615 & 55000 & 6 & 7 \\
& $\sigma_{y}(\mathrm{MPa})$ & 97 & 30 & 10 & 8 \\
\hline Paramètres de viscosité & $K_{\text {visco }}(\mathrm{MPa})$ & 132 & 84 & 8 & 15 \\
\hline \multirow{2}{*}{ Paramètres de l'écrouissage isotrope } & $Q(\mathrm{MPa})$ & 6000 & -85 & 8 & 40 \\
& $b$ & 40 & 0,1 & 25 & 13 \\
\hline \multirow{2}{*}{ Paramètres de l'écrouissage cinématique } & $C(\mathrm{MPa})$ & 42000 & 700 & 16 & 6 \\
& $a$ & 460 & 190 & 30 & 17 \\
\hline \multirow{2}{*}{ Paramètres de l'endommagement du matériau } & $\beta$ & 25 & 6 & 20 & 9 \\
& $S(\mathrm{MPa})$ & 1,15 & 0,55 & 5 & 8,5 \\
& $Y_{0}(\mathrm{MPa})$ & 0,45 & 0 & 20 & 5,5 \\
\hline
\end{tabular}

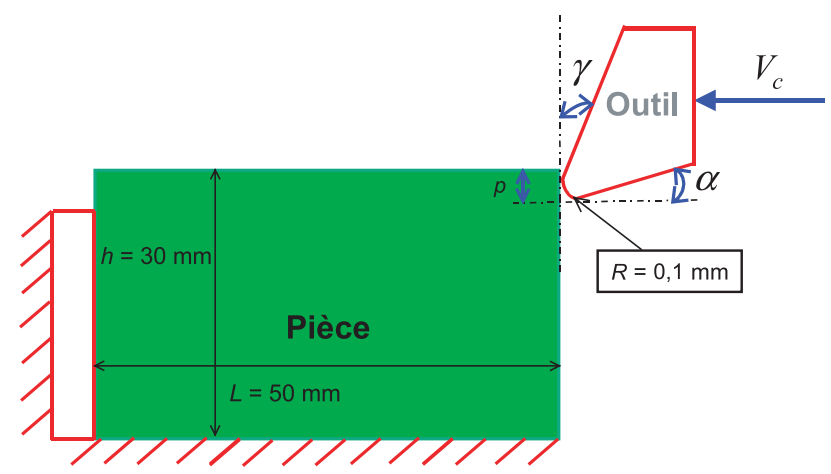

Fig. 1. Usinage en coupe orthogonale.

que la formation du copeau est due à une véritable rupture de la matière devant la pointe de l'outil. Afin de comparer les deux points de vue, nous allons commencer par traiter la formation du copeau sans endommagement. Pour cela nous utilisons notre modélisation sans tenir compte de l'endommagement $\left(k_{\text {couple }}=0\right)$. Pour simplifier la présentation, nous donnons les figures correspondant à un déplacement de l'outil $u=19,345 \mathrm{~mm}$.

\subsubsection{Influence de la profondeur de passe $p$}

Le premier cas traité correspond à l'influence de la profondeur de passe sur la géométrie du copeau ainsi que sur la température. Pour cela, nous avons pris trois profondeurs de passe différentes : $p=0,5 \mathrm{~mm}, p=1 \mathrm{~mm}$, et $p=2 \mathrm{~mm}$. La vitesse d'avance est $V_{\mathrm{c}}=0,05 \mathrm{~mm} . \mathrm{s}^{-1}$, l'angle de coupe est $\gamma=0^{\circ}$ et l'angle de dépouille $\alpha=10^{\circ}$.

La figure 2 illustre la cartographie de la température pour un même déplacement $(u=19,345 \mathrm{~mm})$ à profondeurs de passe différentes. Cette figure montre que la profondeur de passe engendre des différences assez remarquables sur l'enroulement du copeau (Fig. 2). Plus la profondeur de passe est petite plus le copeau s'enroule facilement. Nous voyons aussi que plus la profondeur de passe est grande, plus la température des éléments au contact de la pièce et de l'outil est grande. Lorsque la profondeur de passe est de $2 \mathrm{~mm}$, la température maximale à ce moment-là est de $262,85^{\circ} \mathrm{C}$. Par contre, pour une profondeur de passe de $0,5 \mathrm{~mm}$, elle n'est que de $224,06{ }^{\circ} \mathrm{C}$.

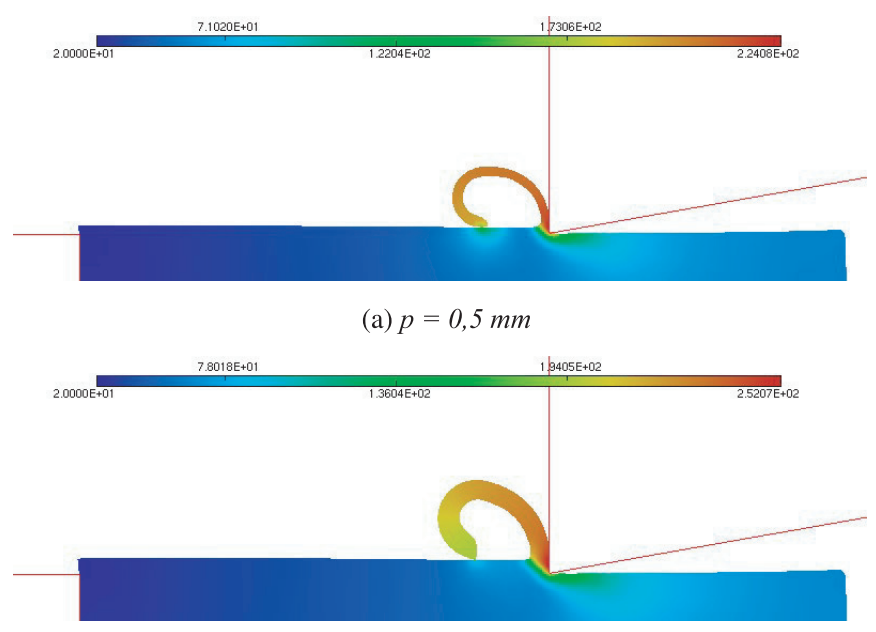

(b) $p=1 \mathrm{~mm}$

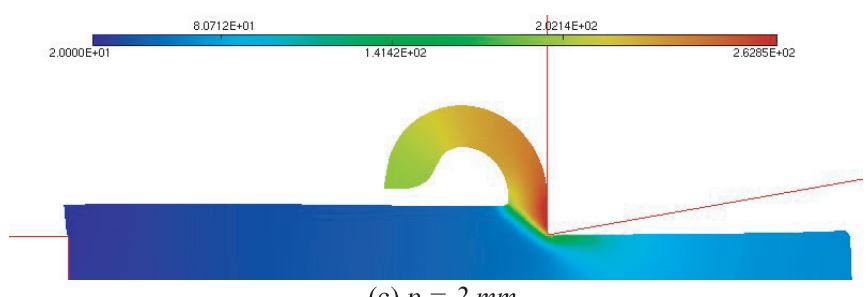

(c) $p=2 \mathrm{~mm}$

Fig. 2. Isovaleurs de la température pour différentes valeurs de la profondeur de passe $(u=19,345 \mathrm{~mm})$.

L'évolution de l'effort de coupe en fonction du déplacement de l'outil est illustrée sur la figure 3. On remarque que plus la profondeur de passe est importante, plus l'effort de coupe est grand. Par exemple, pour un déplacement de l'outil de coupe de $2 \mathrm{~mm}$, il faut une force de $1300 \mathrm{~N}$ pour une profondeur de passe de $2 \mathrm{~mm}$, contre $800 \mathrm{~N}$ pour une profondeur de passe de $1 \mathrm{~mm}$ et $500 \mathrm{~N}$ pour une profondeur de passe de $0,5 \mathrm{~mm}$. Au-delà de ce déplacement, on note une relative stabilisation de l'effort.

\subsubsection{Influence de l'angle de coupe $\gamma$}

L'influence de l'angle de coupe $\gamma$ sur la distribution de la température et de l'effort de coupe est maintenant 


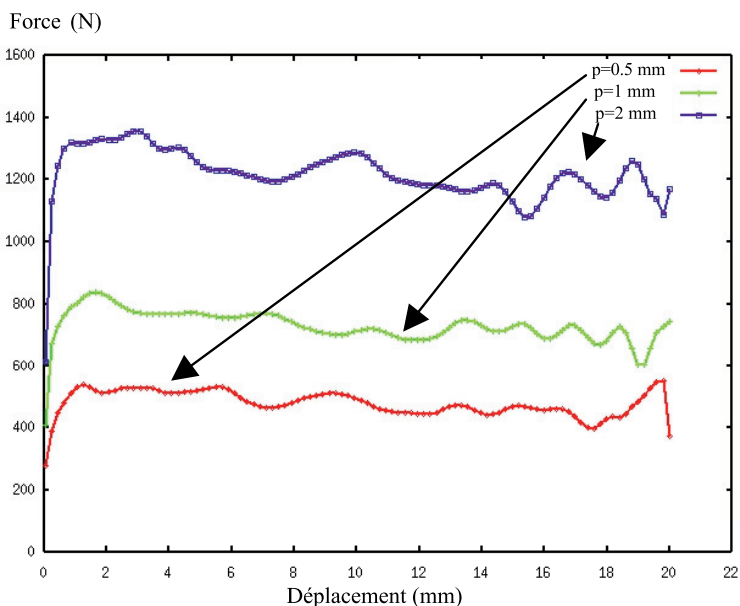

Fig. 3. Courbes force/déplacement pour différentes profondeurs de passe.

examinée. Nous avons fait varier cet angle dans des proportions raisonnables : $0^{\circ}, 5^{\circ}$ et $10^{\circ}$. La vitesse d'avance est $V_{\mathrm{c}}=0,05 \mathrm{~mm} . \mathrm{s}^{-1}$, l'angle de dépouille $\alpha=10^{\circ}$ et la profondeur de passe $p=1 \mathrm{~mm}$.

Nous voyons sur la figure 4 que, suivant l'angle de coupe $\gamma$, la température de la pièce au contact avec l'outil varie. Pour un angle de $10^{\circ}$, la température maximale atteinte est de $227,5{ }^{\circ} \mathrm{C}$ contre $252,07{ }^{\circ} \mathrm{C}$ pour un angle de $0^{\circ}$. Cette différence est essentiellement due à la zone de contact entre la pièce et l'outil : plus elle est importante, plus la température est élevée. Le fait de prendre un grand angle (outil plus coupant) engendre une facilité dans la formation du copeau. Ceci se traduit par un effort maximal de coupe plus faible, comme le montre la figure 5. En effet, la force maximale est de $695 \mathrm{~N}$ pour un angle de $10^{\circ}$ contre $750 \mathrm{~N}$ pour un angle de $0^{\circ}$. On note également une légère différence sur l'enroulement du copeau au profit de l'angle le plus élevé.

\subsubsection{Influence de l'angle de dépouille $\alpha$}

Afin d'étudier l'influence de l'angle de dépouille, nous avons réalisé des essais avec différentes valeurs $\left(0^{\circ}, 5^{\circ}\right.$, $\left.10^{\circ}\right)$. Pour ces différents angles, la vitesse d'avance est de

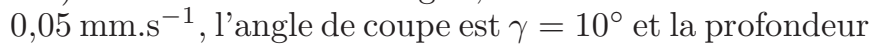
de passe $p=1 \mathrm{~mm}$.

Sur la figure 6 , on remarque que la variation de l'angle de dépouille affecte très peu les résultats. L'enroulement du copeau n'est pas affecté par le changement d'angle. La seule petite différence est la valeur de la température. La température observée pour un angle de $10^{\circ}$ est de $227,58{ }^{\circ} \mathrm{C}$ et celle pour un angle de $0^{\circ}$ est de $214,73^{\circ} \mathrm{C}$. Comparé à l'angle de coupe, pour une même différence d'angle, on avait une variation de température de $25^{\circ} \mathrm{C}$, contre $13{ }^{\circ} \mathrm{C}$, dans ce cas. Il en est de même pour l'effort de coupe, nous observons une influence similaire à celle de l'angle d'attaque $\gamma$ (Fig. 7).

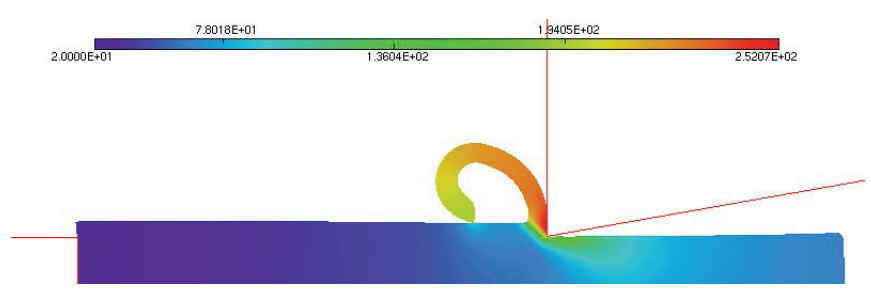

(a) $\gamma=0^{\circ}$

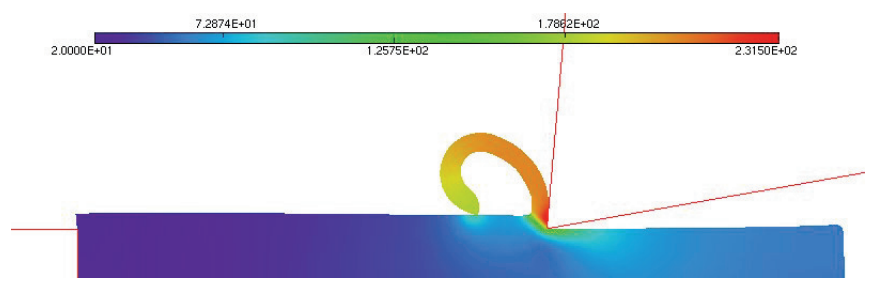

(b) $\gamma=5^{\circ}$

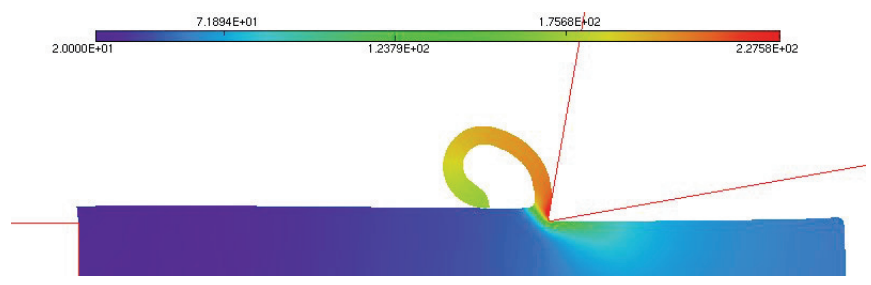

(c) $\gamma=10^{\circ}$

Fig. 4. Isovaleurs de la température pour différentes valeurs de l'angle de coupe $(u=19,345 \mathrm{~mm})$.

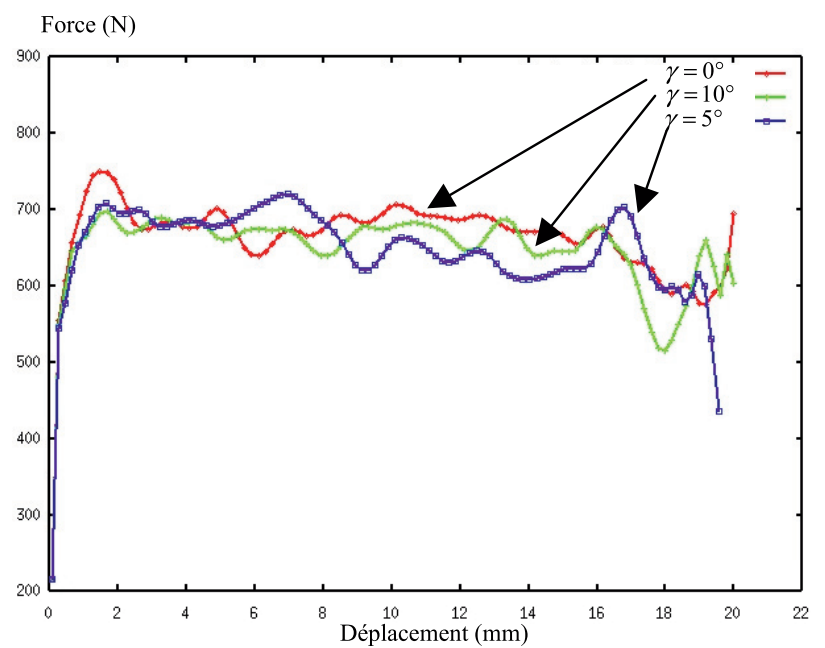

Fig. 5. Courbes force/déplacement pour différents angles de coupe.

\subsubsection{Influence de la vitesse d'avance $V_{c}$}

L'effet de la vitesse de coupe est parmi les paramètres les plus traités en usinage par enlèvement de copeaux. Ainsi, dans ce dernier cas, nous avons fait varier la vitesse de coupe $V_{\mathrm{c}}\left(0,05 \mathrm{~mm} . \mathrm{s}^{-1}, 0,1 \mathrm{~mm} . \mathrm{s}^{-1}, 0,25 \mathrm{~mm} . \mathrm{s}^{-1}\right)$ pour étudier son importance sur la formation du copeau. Nous avons pris un angle de coupe $\gamma=10^{\circ}$, un angle de dépouille $\alpha=10^{\circ}$ et une profondeur de passe de $1 \mathrm{~mm}$. 


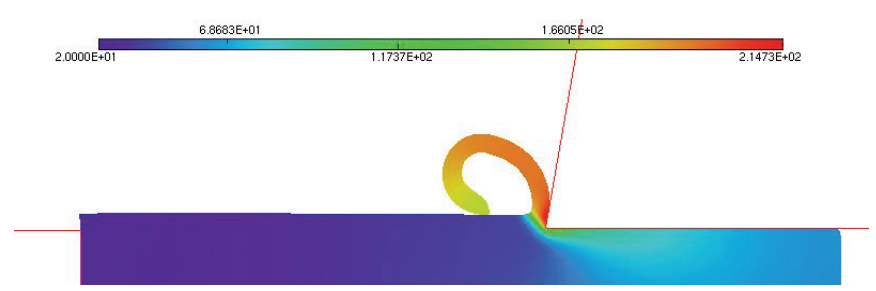

(a) $\alpha=0^{\circ}$

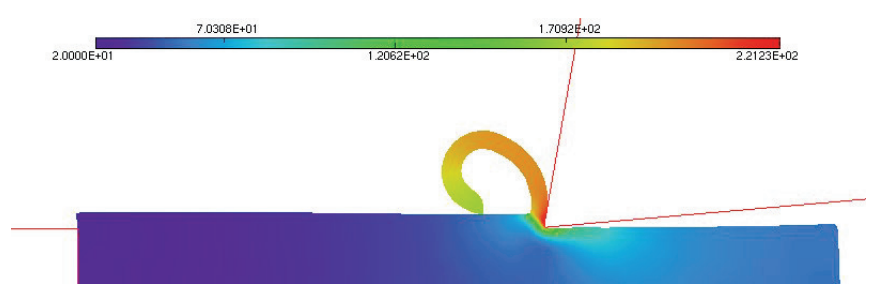

(b) $\alpha=5^{\circ}$

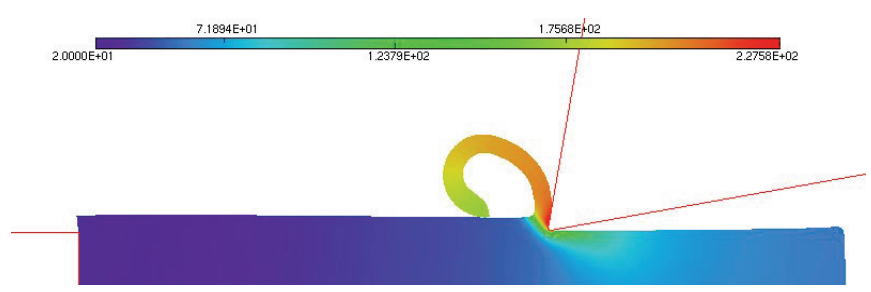

(c) $\alpha=10^{\circ}$

Fig. 6. Isovaleurs de la température pour différentes valeurs de l'angle de dépouille ( $u=19,345 \mathrm{~mm})$.

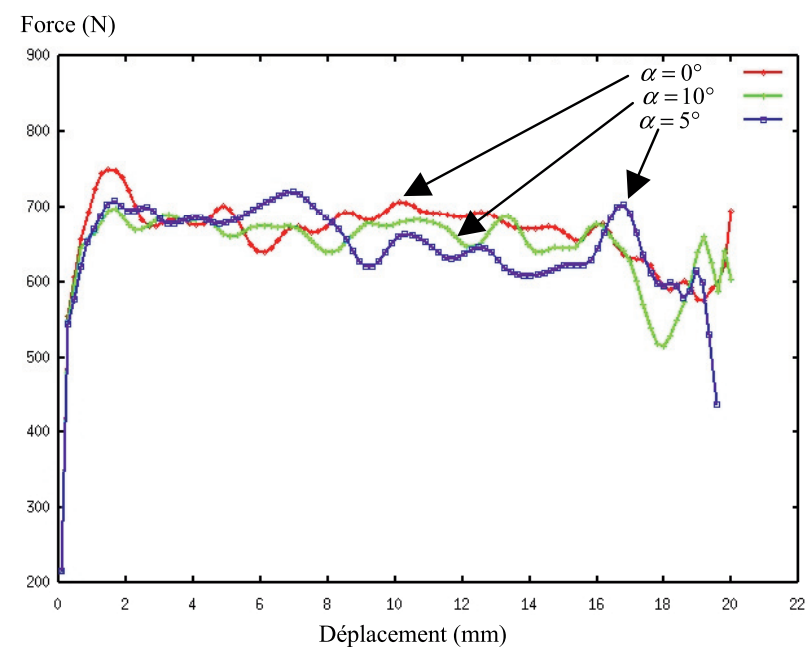

Fig. 7. Courbes force/déplacement pour différents angles de dépouille.

Les isovaleurs de la température pour différentes vitesses de coupe sont données sur la figure 8. On remarque tout de suite que plus la vitesse est grande, plus le copeau reste collé à l'outil. Par exemple, dans le cas où la vitesse de coupe est de $0,25 \mathrm{~mm} . \mathrm{s}^{-1}$, le copeau est resté presque parallèle à l'arête de coupe. De même, plus la vitesse est grande, plus la température est importante. Le copeau restant au contact avec l'arête de coupe, il y a moins d'échange thermique avec le milieu extérieur. La température atteinte lorsqu'on a une vitesse

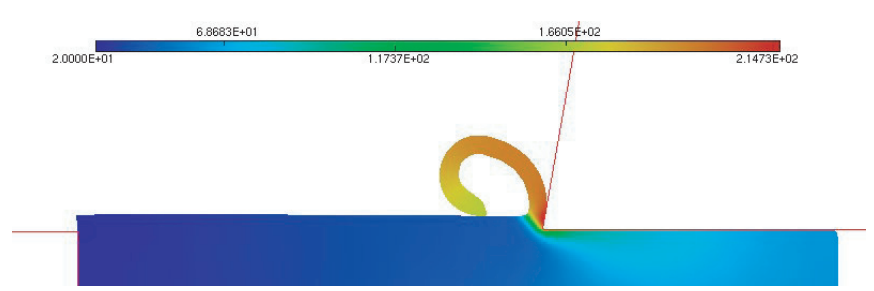

(a) $V_{\mathrm{c}}=0,05 \mathrm{~mm} \cdot \mathrm{s}^{-1}$

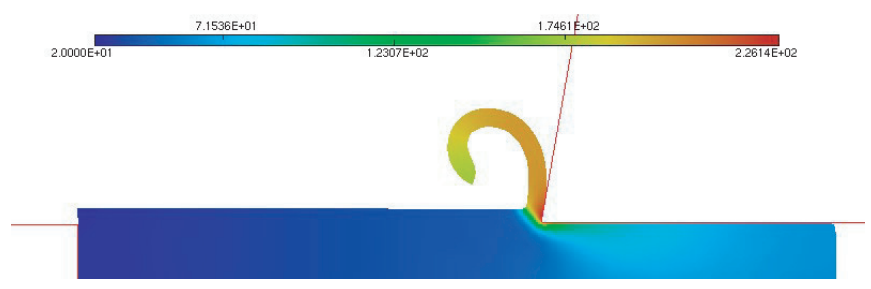

(b) $V_{\mathrm{c}}=0,1 \mathrm{~mm} \cdot \mathrm{s}^{-1}$

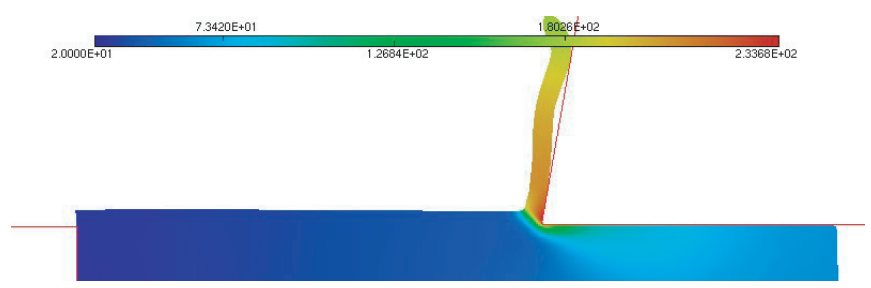

(c) $V_{\mathrm{c}}=0,25 \mathrm{~mm} \cdot \mathrm{s}^{-1}$

Fig. 8. Isovaleurs de la température pour différentes valeurs de la vitesse de coupe $(u=19,345 \mathrm{~mm})$.

de $0,05 \mathrm{~mm} . \mathrm{s}^{-1}$ est de $214,73{ }^{\circ} \mathrm{C}$ (Fig. 8a) alors qu'elle est de $233,68^{\circ} \mathrm{C}$ pour $0,25 \mathrm{~mm} . \mathrm{s}^{-1}$.

L'évolution de l'effort de coupe en fonction de l'avance de l'outil est donnée sur la figure 9 pour différentes vitesses de coupe $V_{\mathrm{c}}$. On peut remarquer que l'augmentation de la vitesse de coupe engendre une légère croissance de l'effort de coupe (Fig. 9). Pour une vitesse importante $V_{\mathrm{c}}=0,25 \mathrm{~mm} . \mathrm{s}^{-1}$ la force est de l'ordre de $770 \mathrm{~N}$. Par contre, pour une vitesse plus faible de $0,05 \mathrm{~mm} . \mathrm{s}^{-1}$, la force maximale est de $700 \mathrm{~N}$.

Nous notons enfin que le copeau formé par cette méthode est un copeau continu sans aucune possibilité de décrire sa fragmentation. Cependant dans la pratique on observe souvent une fragmentation du copeau. Cette fragmentation est très dépendante de la ductilité du matériau et des conditions de coupe si l'on néglige les problèmes de vibration (broutement).

\subsection{Calculs avec couplage comportement-endommagement}

Nous allons maintenant refaire l'étude ci-dessus en utilisant la méthodologie tenant compte du couplage comportement-endommagement. Pour étudier le phénomène de fragmentation du copeau, nous ferons varier la ductilité du matériau de 1,65\% (matériau fragile) à 18,9\% (matériau ductile). Ceci est gouverné par le paramètre $S$ du modèle d'endommagement qui varie alors de 1,15 à $8,15 \mathrm{MPa}$ (Tab. 3). Tous les autres paramètres du 


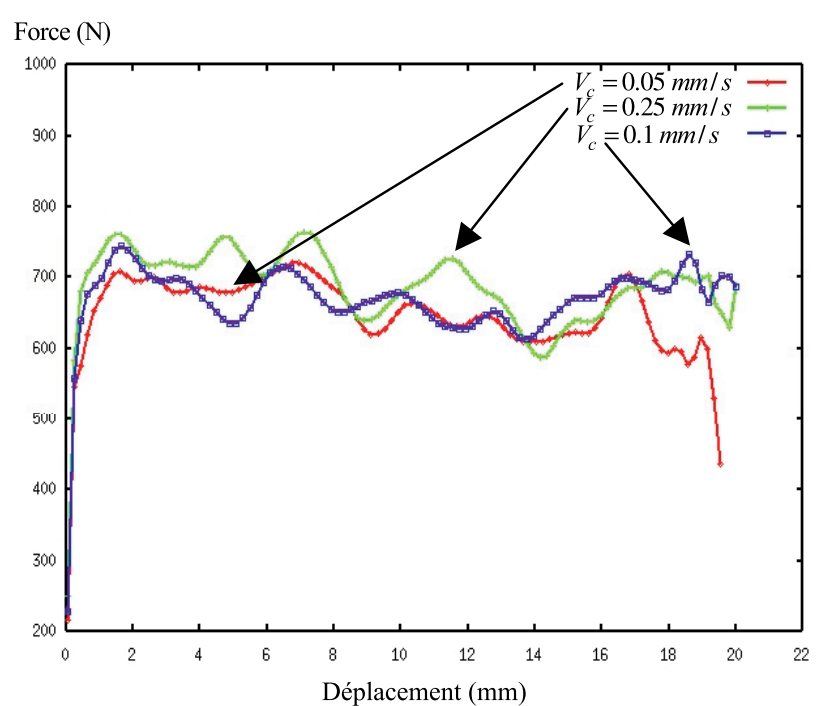

Fig. 9. Courbes force/déplacement pour différentes vitesses de coupe.

Tableau 3. Propriétés du matériau pour différentes ductilités.

\begin{tabular}{cc}
\hline $\begin{array}{c}\text { Coefficient d'endommagement } S \\
\text { en } \mathrm{MPa}\end{array}$ & $\begin{array}{c}\text { Déformation } \\
\text { à la rupture }\end{array}$ \\
\hline 1,15 & $1,65 \%$ \\
4,15 & $11,14 \%$ \\
5,15 & $14 \%$ \\
8,15 & $18,9 \%$ \\
\hline
\end{tabular}

matériau sont les mêmes que pour la série de simulation précédente avec le modèle non couplé.

La taille des éléments est toujours $h_{\min }=0,2 \mathrm{~mm}$ et $h_{\max }=4 \mathrm{~mm}$. Les outils sont supposés rigides. Les mêmes conditions expérimentales ont été utilisées dans cette simulation : c'est-à-dire un outil possédant un angle de dépouille $\alpha=0^{\circ}$ et un angle de coupe $\gamma=10^{\circ}$. La profondeur de passe prise est $p=1 \mathrm{~mm}$ et une vitesse d'avance de l'outil $V_{\mathrm{c}}=0,05 \mathrm{~mm} . \mathrm{s}^{-1}$. Sur la figure 10 sont représentées les isovaleurs de la température pour différentes ductilités des matériaux pour un déplacement de l'outil de coupe de 4,99 $\mathrm{mm}$.

Suivant la ductilité du matériau, la distribution de température est totalement différente. Plus la ductilité est grande plus la température est élevée dans le copeau comme on peut s'y attendre. La température passe de $207{ }^{\circ} \mathrm{C}$ pour matériau le plus ductile à $22,4{ }^{\circ} \mathrm{C}$ pour le matériau le moins ductile. On note également une forte segmentation du copeau pour le matériau le plus fragile que nous allons interpréter en détails. Concernant la formation et la segmentation du copeau, elle est bien décrite par le modèle, comme le montrent la figure 11 et les suivantes.

Pour le matériau le plus ductile (déformation à la rupture de $18,9 \%$ ), le copeau se forme, se propage, puis se segmente pour un déplacement de l'outil de 20,49 mm comme le montre la figure 11 où l'on a reporté les distributions de la température (Fig. 11a),

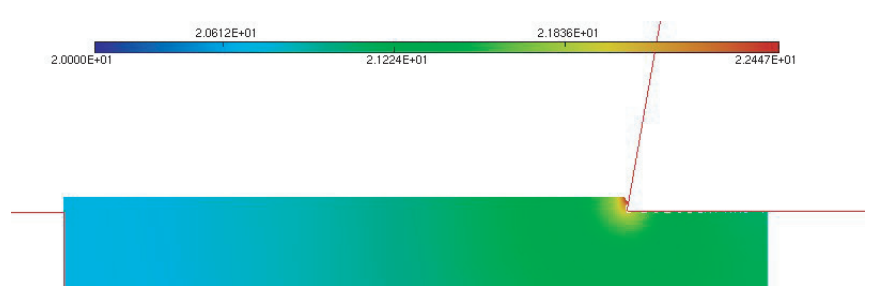

(a) $S=1,15 \mathrm{MPa}$ (ductilité de 1,65\%)

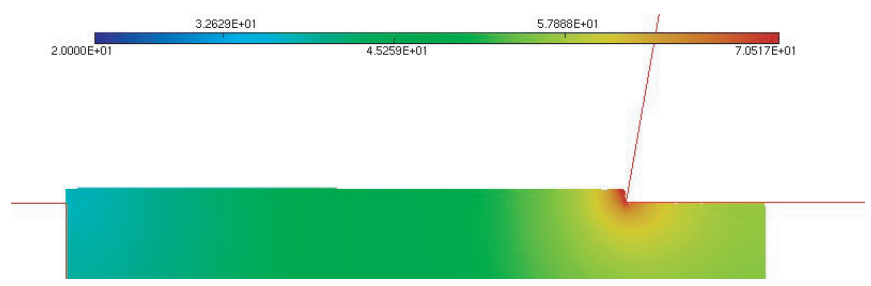

(b) $S=4,15 \mathrm{MPa}$ (ductilité de $11,14 \%$ )

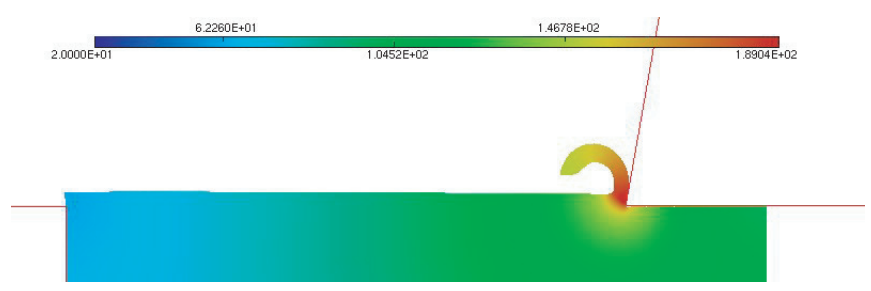

(c) $S=5,15 \mathrm{MPa}$ (ductilité de $14 \%$ )

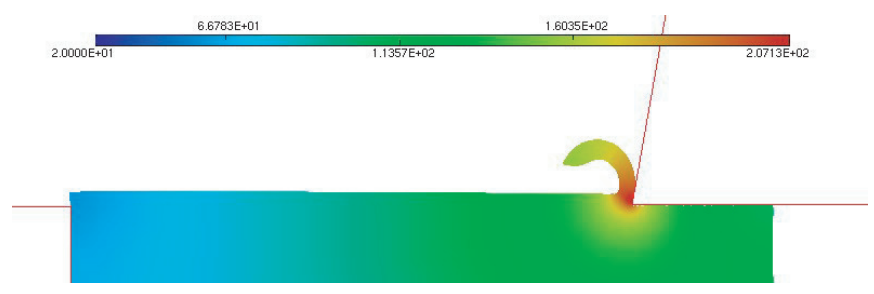

(d) $S=8,15 \mathrm{MPa}$ (ductilité de 18,9\%)

Fig. 10. Isovaleurs de la température pour différentes ductilités.

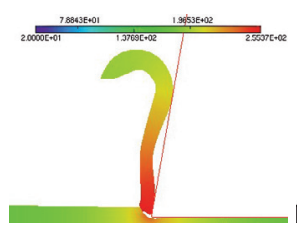

a) Température

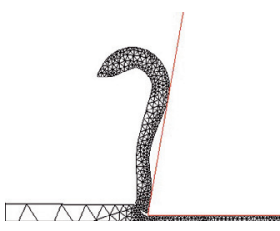

b) Maillage

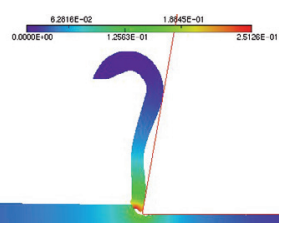

c) Endommagement
Fig. 11. $S=8,15 \mathrm{MPa}$ : déplacement $u=20,49 \mathrm{~mm}$.

l'adaptation du maillage (Fig. 11b) ainsi que l'endommagement (Fig. 11c).

À cet instant, la température maximale est de $255^{\circ} \mathrm{C}$ et la « cassure »du copeau est achevée. Le premier segment de copeau a alors une taille de 20,5 mm.

Pour le matériau ayant $14 \%$ de ductilité, la situation est assez différente, comme le montrent les figures 12 et 13. Le copeau se forme et se segmente à une longueur de 15,29 mm (Fig. 12). 


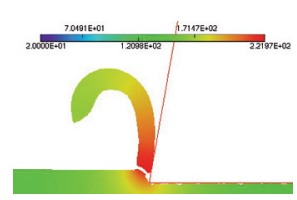

a) Température

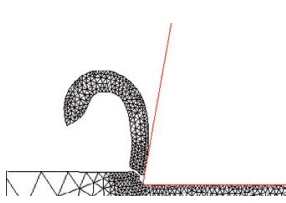

b) Maillage

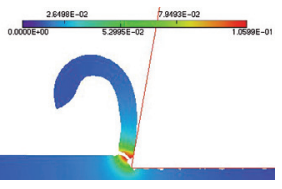

c) Endommagement
Fig. 12. $S=5,15 \mathrm{MPa}$ : déplacement $u=15,29 \mathrm{~mm}$.

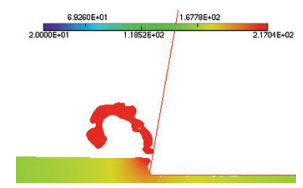

a) Température

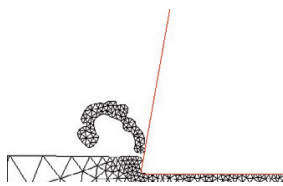

b) Maillage

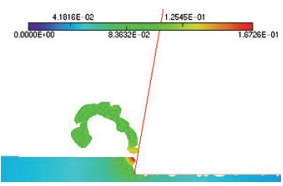

c) Endommagement
Fig. 13. $S=5,15 \mathrm{MPa}$ : déplacement $u=23,09 \mathrm{~mm}$.

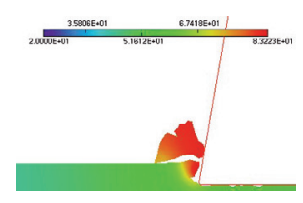

a) Température

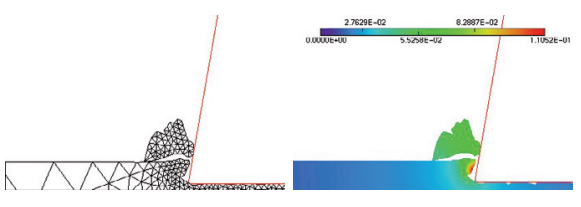

b) Maillage

c) Endommagement
Fig. 14. $S=4,15 \mathrm{MPa}$ : déplacement $u=4,74 \mathrm{~mm}$.

La température maximale à cet instant est de $222^{\circ} \mathrm{C}$. On note que la baisse de ductilité provoque une baisse de la longueur du premier fragment de copeau. Si on continue l'usinage, un deuxième copeau se forme et se segmente pour un déplacement de l'outil de 23,09 $\mathrm{mm}$, donnant une longueur de ce deuxième fragment de copeau de 7,8 mm contre $15,29 \mathrm{~mm}$ pour le premier fragment.

On note aussi l'existence de plusieurs fissures transversales sur ce deuxième fragment lui donnant ainsi une géométrie en dents de scie souvent observée expérimentalement. Enfin notons que la température maximale à la formation du deuxième fragment est de $217^{\circ} \mathrm{C}$.

Pour le matériau à 11,14\% de ductilité, le premier fragment de copeau a une longueur de $4,74 \mathrm{~mm}$ et contient quelques fissures transversales comme on peut le voir sur la figure 14 .

Les fragments suivants ne sont même plus visibles car leur longueur est inférieure à $1 \mathrm{~mm}$ et la taille du maillage $\left(h_{\min }=0,2 \mathrm{~mm}\right)$ ne permet plus de les visualiser. En effet, pour cela, il aurait fallu prendre un $h_{\min }$ plus faible de l'ordre de 0,01 mm, mais cela nécessitait une nouvelle identification. Quant à la température maximale, elle est de l'ordre de $83,2{ }^{\circ} \mathrm{C}$ au moment de la formation du premier fragment.

Enfin, nous terminons par le matériau le moins ductile (déformation à la rupture de 1,65\%). Les fragments du copeau (y compris le premier) sont de l'ordre de $0,2 \mathrm{~mm}$ et il n'est pas possible de les visualiser pour les mêmes raisons que précédemment. De ce fait, la température maximale après un déplacement de l'outil de 4,99 $\mathrm{mm}$ est de l'ordre de $22,4^{\circ} \mathrm{C}$ (Fig. 10a).

Pour conclure sur l'effet de la ductilité, on peut noter que plus le matériau est ductile plus la longueur du

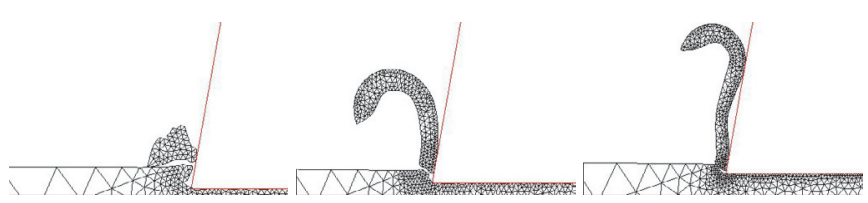
a) $S=4,15 \mathrm{MPa}$
b) $S=5,15 \mathrm{MPa}$
c) $S=8,15 \mathrm{MPa}$

Fig. 15. Maillage à la première segmentation du copeau pour différentes ductilités.

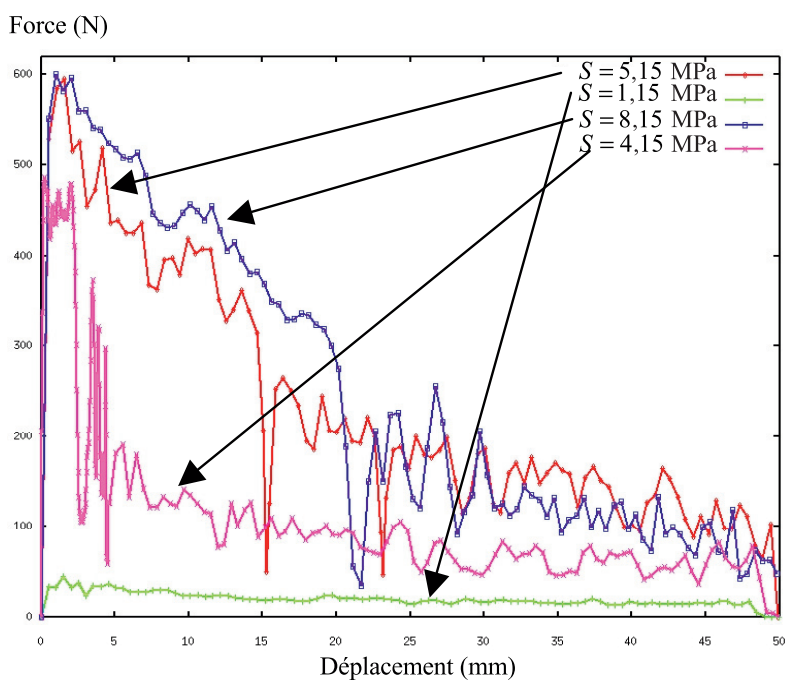

Fig. 16. Courbes force/déplacement pour différentes ductilités.

premier fragment de copeau est grande (Fig. 15) et plus la température est naturellement plus élevée.

On s'attend donc que, pour une ductilité suffisamment grande, le copeau ne se segmente jamais, donnant un copeau continu en forme de spirale conformément aux observations expérimentales. L'autre remarque est que les fragments de copeaux sont d'autant plus courts que leur nombre est élevé. L'ensemble de ces phénomènes captés par l'approche avec endommagement ne sont pas « captables » par l'approche sans endommagement.

Examinons maintenant l'allure des courbes force/déplacement pendant les opérations d'usinage et ce pour différentes ductilités (Fig. 16). L'allure typique de ces courbes est la suivante : l'effort augmente rapidement pour atteindre un maximum et amorce une chute « continue» au fur et à mesure que le copeau se forme. À la séparation du premier fragment de copeau, l'effort chute brutalement puis remonte rapidement pour amorcer le nouveau fragment de copeau et ainsi de suite. En exemple, prenons le cas du matériau avec $14 \%$ de ductilité $(S=5,15 \mathrm{MPa})$. L'effort maximal est de $590 \mathrm{~N}$ puis diminue progressivement. À $u=15,29 \mathrm{~mm}$ (c'est-à-dire à la séparation du premier fragment de copeau), l'effort chute brutalement pour atteindre $55 \mathrm{~N}$ et remonte aussitôt à $250 \mathrm{~N}$ pour amorcer le deuxième fragment de copeau. L'effort amorce alors une chute continue pendant la progression du deuxième fragment qui se détache pour $u=23,09 \mathrm{~mm}$ où l'effort s'effondre de nouveau, ainsi de suite. 


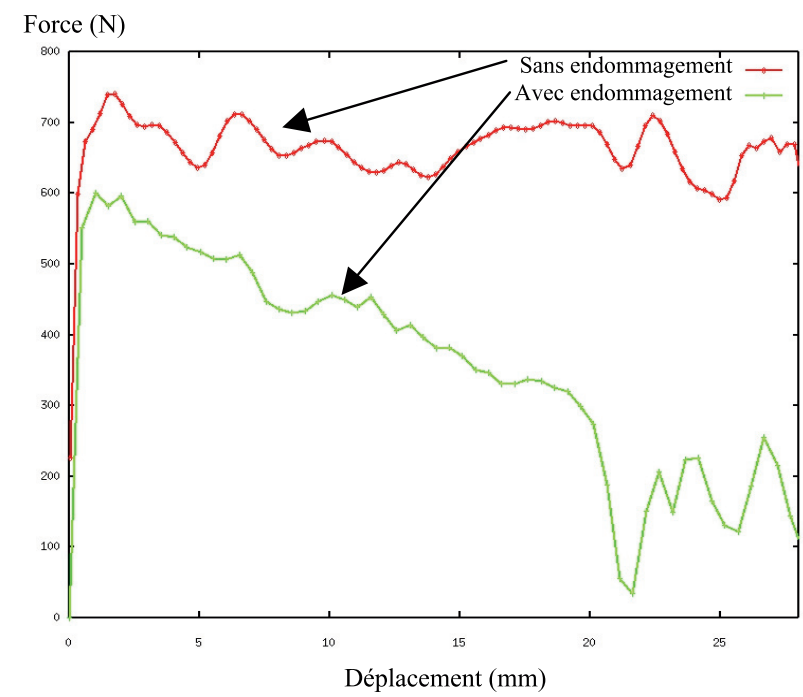

Fig. 17. Courbes force/déplacement avec ou sans endommagement.

Pour le matériau le moins ductile (déformation à la rupture de 1,65\%), l'effort de coupe est très faible $(<40 \mathrm{~N})$ et sa variation est plus lisse à cause des faibles tailles de fragments qui se forment avec une grande facilité, nécessitant un faible effort. Enfin, on note que l'effort de coupe nécessaire à la formation du premier copeau est d'autant plus élevé que le matériau est ductile.

Sur la figure 17, nous comparons l'effort de coupe pour le matériau le plus ductile avec le calcul couplé (avec l'endommagement) et non couplé (sans endommagement). Deux remarques retiennent l'attention :

- L'effort de coupe est nettement plus élevé avec l'approche sans endommagement, il est supérieur à $700 \mathrm{~N}$ contre $600 \mathrm{~N}$ dans l'approche avec endommagement.

- L'effort oscille autour d'une valeur constante tout le temps pour l'approche sans endommagement. Dans le cas avec endommagement, on note une chute de l'effort de $595 \mathrm{~N}$ à environ $320 \mathrm{~N}$ au moment de la fragmentation du premier copeau puis chute à $30 \mathrm{~N}$ lors de la séparation du premier fragment $(u=20,5 \mathrm{~mm})$ pour remonter immédiatement.

En conclusion, l'ensemble des résultats discutés cidessus montre que si la formation du copeau est modélisable avec ou sans endommagement, la segmentation du copeau n'est possible qu'avec l'approche tenant compte de l'endommagement ductile. De plus, cette dernière semble plus apte que l'approche sans endommagement à rendre compte de plusieurs phénomènes caractéristiques de la coupe par formation de copeaux ou d'autre type de coupe comme le refendage des tôles, l'estampage, le matriçage, ...

Une dernière comparaison entre ces deux approches consiste à pousser l'usinage jusqu'à la fin de la pièce afin d'examiner la séparation finale pièce-copeau. Pour cela, nous avons traité l'usinage d'une pièce de $10 \mathrm{~mm}$ de longueur en utilisant les mêmes conditions que cidessus en supposant un matériau à $14 \%$ de ductilité

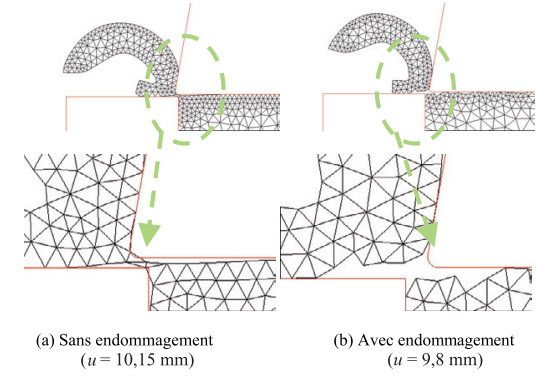

Fig. 18. Maillage pour un calcul sans endommagement (déplacement 10,15 $\mathrm{mm}$ ) et pour un calcul avec endommagement (déplacement 9,8 $\mathrm{mm}$ ).

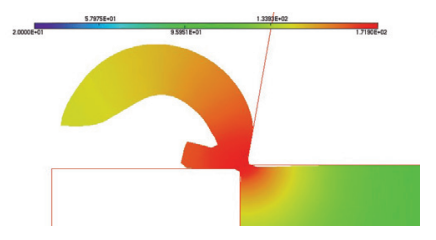

(a) Sans endommagement

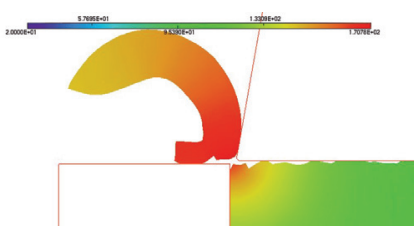

(b) Avec endommagement
Fig. 19. Isovaleurs de la température pour un déplacement $u=9,8 \mathrm{~mm}$.

$(S=5,15 \mathrm{MPa})$. Dans les deux cas, on observe bien une formation de copeaux jusqu'au bout de la pièce (Fig. 18).

La principale différence se situe dans la séparation entre la pièce et le copeau. Pour l'approche couplée, la séparation finale pièce-copeau est bien décrite (Fig. 18b), alors que pour l'approche sans endommagement, un ligament de matière demeure (Fig. 18a). Les fortes distorsions du maillage dans ce ligament provoque l'arrêt du calcul. Sur le plan thermique, il n'y a pas de différence significative entre la distribution de la température dans les deux cas, comme le montre la figure 19.

\section{Conclusion}

En traitant l'exemple de la coupe orthogonale avec deux approches, l'une tenant compte de l'endommagement ductile et de son effet sur les autres champs thermo-mécaniques (couplages forts) et l'autre négligeant totalement la notion d'endommagement, nous pouvons affirmer que :

- L'une et l'autre des méthodes permettent de simuler la formation du copeau.

- Seule l'approche avec endommagement rend compte naturellement et d'une manière réaliste de la segmentation du copeau.

- Seule l'approche avec endommagement modélise correctement la séparation copeau-pièce à la fin de l'usinage orthogonal.

- L'allure des courbes effort de coupe déplacement de l'outil est correctement décrite par l'approche avec endommagement.

- L'approche couplée traduit bien la fragmentation du copeau en fonction de la ductilité du matériau. 
- L'influence des paramètres du procédé (vitesse de coupe, profondeur de passe, ... ) est qualitativement bien décrite par les deux approches.

Ceci semble indiquer que l'approche tenant compte $\mathrm{du}$ couplage comportement-endommagement possède d'intéressantes potentialités pour simuler numériquement la coupe par formation de copeaux. Ceci dit, trois aspects nous semblent importants à améliorer dans cette approche.

- La dépendance de la solution vis-à-vis de la finesse du maillage dans les zones endommagées. Cela nécessite l'utilisation d'une théorie non locale.

- Améliorer le couplage frottement-thermique afin que la chaleur générée par le frottement copeau-outil et pièce-outil soit prise en compte dans le bilan énergétique.

- Améliorer le modèle mécanique afin d'y inclure les « vrais » mécaniques physiques de formation du copeau. En particulier tenir compte des phénomènes métallurgiques (voir chimiques) se produisant à l'interface outil copeau afin de modéliser des phénomènes comme l'usure de l'outil, avec formation de cratères sur la face de coupe ou avec l'adhésion de copeaux à la surface de coupe ...

Ceci demande bien entendu de disposer de mesures expérimentales pertinentes qui ne semblent pas facilement disponibles à l'heure actuelle.

Remerciements. Nous remercions le soutien financier de la région Champagne-Ardenne (CRCA) et de l'état (FEDER contrat $n^{\circ}$ 99-2-50-059) dans le cadre du Pôle Mécanique et Matériaux Champardennais (PMMC). Les auteurs remercient également le Professeur H. Borouchaki du LASMIS pour l'utilisation du mailleur BL2D-V2.

\section{Références}

[1] A. Firas, Modélisation et simulation thermomécaniques de la coupe des métaux, Thèse de doctorat, ENSAM, Paris, 2001
[2] J.D. Kim, V.R. Marinov, D.S. Kim, Built-up edge analysis of orthogonal cutting by the visco-plastic finite element method, J. Mat. Proc. Tech. 71 (1997) 367-372

[3] R. Rakotomalala, P. Joyot, M. Touratier, Communication in numerical methods in engineering 9 (1993) 975-987

[4] Z.C. Lin, S.Y. Lin, A coupled finite element model of thermo-elastic-plastic large deformation for orthogonal cutting, J. Eng. Mater. Technol. 114 (1992) 218-226

[5] A.G. Mamalis, M. Horvath, A.S. Branis, D.E. Manolakos, Finite element simulation of chip formation in orthogonal metal cutting, J. Mat. Proc. Tech. 110 (2001) 19-27

[6] K. Komvopoulos, S.A. Erpenbeck, Finite element modeling of orthogonal metal cutting, Trans. ASME J. Eng. For. Ind. 113 (1991) 253-267

[7] E. Ceretti, P. Fallboehmer, W.T. Wu, T. Altan, Simulation of high speed milling: application of 2D FEM to chip formation in orthogonal cutting, ERC NSM, Ohio State University, 1995

[8] L. Fourment, P.O. Bouchard, Numerical simulation of chip formation and crack propagation during non-steady cutting processes, Int. J. Forming processes (Modeling of Machining Operations) 3(1-2) (2000) 59-76

[9] P. Lestriez, Modélisation numérique du couplage thermomécanique-endommagement en transformations finies, Application à la mise en forme, Thèse de doctorat, UTT, Troyes, 2003

[10] Y. Zhu, Contribution to the local approach of fracture in solid dynamics, Liège, 1995

[11] J. Lemaitre, J.L. Chaboche, Mécanique des Matériaux Solides, Dunod, 1985

[12] K. Saanouni, J.L. Chaboche, Computational Damage Mechanics. Application to Metal Forming, Chapter 7 of the Volume 3: Numerical and Computational methods, R. de Borst, H.A. Mang (ed.), in Comprehencive Structural Integrity, edited by I. Milne, R.O. Ritchie, B. Karihaloo, ISBN : 0-08-043749-4, 2003

[13] H. Borouchaki, P. Laug, BL2D-V2 : mailleur bidimensionnel adaptatif, Rapport $\mathrm{n}^{\circ} 0275$ INRIA, 2003

[14] H. Borouchaki, P. Laug, A. Cherouat, K. Saanouni, Adaptative remeshing for ductile fracture prediction in metal forming, C.R. Acad. Sci. 330(10) (2002) 709-716

[15] A. Elhraiech, Simulation numérique de la coupe orthogonale : Application à l'alliage d'aluminium AS7U3G.T5, DEA, Université de Technologie de Troyes, 2003 